\title{
A comparative study between placental alpha microglobulin-1 rapid immunoassay and standard diagnostic methods for detection of rupture of membranes
}

\author{
Hemant Deshpande, Umesh Sabale*, C. S. Madkar, Anuja Bobe
}

Department of Obstetrics and Gynecology, Dr. D. Y. Patil Medical College, D. Y. Patil Vidyapeeth, Pune, Maharashtra, India

Received: 21 February 2018

Accepted: 27 March 2018

\section{*Correspondence:}

Dr. Umesh Suresh Sabale,

E-mail: drumeshsabale@gmail.com

Copyright: $\odot$ the author(s), publisher and licensee Medip Academy. This is an open-access article distributed under the terms of the Creative Commons Attribution Non-Commercial License, which permits unrestricted non-commercial use, distribution, and reproduction in any medium, provided the original work is properly cited.

\section{ABSTRACT}

Background: To determine the efficacy of an immunoassay to measure levels of placental alpha-microglobulin-1 in cervico-vaginal secretions.

Methods: 100 ANC cases admitted in Dr D. Y. Patil Hospital with symptoms of rupture of membranes during study period. Inclusion criteria-Pregnant women who presented with symptoms of ROM either in labour or not in labour, gestational age from 28 weeks onwards and who have given consent.

Results: In 9 cases, the immunoassay test was negative, in 91 cases, it was positive and in one case, the liquor had a lot of meconium, the woman had to undergo an emergency cesarean section. This patient was deemed to have a false negative result by the test. Pooling was positive in 87 cases, nitrazine test in 86 cases and ferning in 88 cases. Hence, in comparison to immunoassay test which had $100 \%$ specificity and $98.91 \%$ sensitivity due to incomplete evaluation, nitrazine test had $95.58 \%$ sensitivity and $100 \%$ specificity, Pooling has $97.79 \%$ sensitivity and $100 \%$ specificity, whereas ferning has $98.34 \%$ sensitivity and $100 \%$ specificity.

Conclusions: The PAMG1 is a non-invasive, rapid, one step test with very high sensitivity, specificity and accuracy as compared to the conventional methods together and also individually. Preforming this test instead of conventional methods can aid the early detection of rupture of membranes and largely affect the outcome of maternal and fetal health as timely decision can be taken once diagnosis is confirmed.

Keywords: Ferning test, Immunoassay, Nitrazine test, Placental alpha microglobulin-1, Premature rupture of membranes (PROM)

\section{INTRODUCTION}

Premature rupture of membranes (PROM), defined as spontaneous rupture of the fetal membranes before the onset of uterine contractions, is one of the most common diagnostic dilemmas in contemporary obstetric practice. PROM can occur at any gestational age, and preterm PROM (defined as PROM before 37 weeks) is responsible for $20-40 \%$ of preterm births. ${ }^{1}$ It is the leading identifiable cause of premature birth and accounts for approximately $18 \%$ to $20 \%$ of perinatal deaths.
According to Lui et al, Lancet 2012 report, prematurity and low birth weight accounts for $35 \%$ of total neonatal deaths in India. So early and accurate diagnosis of PROM would allow for gestational age-specific obstetric interventions designed to optimize perinatal outcome and minimize serious complications.

Since there is significant incidence of PROM and PPROM in pregnancies, it is necessary to have good methods for diagnosis as a positive result will help tackle the complications at the right time and a negative result 
will help avoid unnecessary intervention; both physical and operative and allow the pregnancy to continue till term in cases of PPROM.

Conventional clinical methods for diagnosing ROM are seriously flawed. Three commonly used methods for confirming rupture of membranes (ROM) are obvious leakage of fluid from the cervix, positive nitrazine test, and ferning.

Neither of these tests have been found to be $100 \%$ sensitive or specific.

A bedside immunoassay designed to measure levels of the $34 \mathrm{kd}$ fetal glycoprotein, placental alphamicroglobulin-1, in cervicovaginal secretions was recently introduced into clinical practice in an attempt to improve on conventional clinical tests. ${ }^{3}$ Placental alphamicroglobulin-1 (PAMG-1) is considered an ideal candidate marker for ROM because its concentration in amniotic fluid is 1,000- to 10,000-fold higher than that in the cervicovaginal discharge with intact membranes (2,000-25,000 $\mathrm{ng} / \mathrm{mL}$ versus $0.05-2.0 \mathrm{ng} / \mathrm{mL}$, respectively). ${ }^{4}$ It is also not present in urine or semen, and at low levels in maternal blood, reducing the risk of inaccurate results in the presence of other fluids.

This immunochormatographic assay is an easy, one-step, bedside test. The test employs highly sensitive and specific proprietary monoclonal antibodies (MAB) that detect even a minimum amount of the amniotic fluid protein, PAMG-1. The test consists of a swab stick, a solvent and a strip and it takes about 5-10 minutes to carry out.

This study is to compare the diagnostic accuracy of this immunoassay with that of conventional standard diagnostic methods in diagnosing ROM. The standard diagnostic method of diagnosing rupture of membrane is defined as positive for two of the following three clinical signs:

- Visualisation of fluid pooling in the posterior fornix,

- Positive ferning test,

- Positive nitrazine test.

The Objectives of the present were to determine the efficacy of an immunoassay to measure levels of placentalalpha-microglobulin-1 in cervico-vaginal secretions and to compare the accuracy of an immunoassay to measure levels of placental alphamicroglobulin-1 with that of standard diagnostic methods (clinical examination, nitrazine test and fern test) for the diagnosis of rupture of membranes.

\section{METHODS}

It was a prospective observational study performed in 100 consecutive patients with signs or symptoms of rupture of membranes at Dr. D. Y. Patil Medical College, Hospital and Research Centre, Pimpri, Pune from July 2014 to February 2016.

Pregnant women who presented with symptoms of ROM either in labour or not in labour, gestational age from 28 weeks onwards, and those who will give consent are included. We excluded pregnant women with active vaginal bleeding, those diagnosed to have placenta praevia and those with intrauterine death. The study was approved by the hospital's Institutional scientific review Board and ethical committee. Written and informed consent was obtained from all patients.

A diagnosis of ROM will be made if two of the following three clinical signs will be present:

- Visual pooling of fluid in the posterior fornix

- Positive nitrazine test

- Microscopic evidence of ferning.

Performing the Immunoassay test on patients while performing clinical examination.All eligible patients will be evaluated for ROM with a detailed history, physical examination (including sterile speculum examinations), and ultrasound examination. At initial speculum examination without the use of antiseptic solutions, all patients will be assessed using both conventional clinical tests -visual evaluation of leakage or pooling of amniotic fluid in the posterior fornix, nitrazine testing, and ferning, and the Placental alpha-microglobulin-1 immunoassay. Women will be included in the study only at their initial presentation. The monoclonal antibodies (MABs- A and B) employed in the test have been specifically selected to recognise $5 \mathrm{ng} / \mathrm{ml}$ of PAMG-1 molecules in the vaginal discharge of the pregnant woman. PAMG-1 rapid immunoassay test was performed by placing a sterile Dacron swab at the posterior vaginal fornix for about one minute in a noninvasive way to collect a sample of vaginal discharge. The swab is then washed in the vial with solvent that extracts the sample substance from the swab. Finally, the pad portion of the test strip is inserted into the vial and the result is read in minutes. A-MABs in the soluble form are located in the pad region. These MABs have colloidal gold particles attached to them. When A-antibodies come in contact with PAMG-1 protein, resulting from the ROM and the subsequent leakage of the amniotic fluid, they catch PAMG-1 and transport it to the test region. The test region of the test strip has B-antibodies immobilized on it. B-antibodies meet PAMG-1 bound to A-MABs flowing up from the pad region. This meeting immobilizes the system of PAMG-1/A-MABs, resulting in a brown/yellow stripe that becomes visible in the test region. This stripe is produced by dye gold attached to A-antibodies and indicates a rupture of fetal membranes.

\section{RESULTS}

In 9 cases, the immunoassay test was negative, in 91 cases, it was positive \& in one case, the liquor had a lot 
of meconium, the woman had to undergo an emergency cesarean section. This patient was deemed to have a false negative result by the test.

Table 1: Association between Fern test/Nitrazine Test/Pooling of liquor (Any two-test positive) and PAMG1 Immunoassay Test in study groups.

\begin{tabular}{|llll|}
\hline PAMG1 & \multicolumn{2}{l}{$\begin{array}{l}\text { Fern test/Nitrazine } \\
\text { immunoassay test }\end{array}$} & \multicolumn{2}{l|}{ Test/Pooling of liquor } \\
Positive & Negative & Total \\
\cline { 2 - 3 } Positive & 91 & 0 & 91 \\
\hline Negative & 1 & 8 & 9 \\
\hline Total & 92 & 8 & 100 \\
\hline
\end{tabular}

Fisher exact test: $\mathrm{P}<0.0001$, Sensitivity $=98.91 \%$, Specificity $=100 \%$, $\mathrm{PPV}=100 \%, \mathrm{NPV}=88.89$, Accuracy $=99 \%$

If we compare the association between the conventional clinical methods (performing all 3 and where any 2 are positive) and PAMG1 Immunoassay, the results are incredible with sensitivity of $98.91 \%$, specificity of $100 \%$ and accuracy of $99 \%$. Also, the $\mathrm{P}$ value calculated by Fisher test is $<0.0001$ which is statistically highly significant.

Table 2: Association between leucocytosis and PAMG1 Immunoassay Test in study group.

\begin{tabular}{|lll|l|} 
Leucocytosis & PAMG1 Immunoassay & Total \\
\cline { 2 - 4 } & Positive & Negative & \\
\hline Present & 60 & 0 & 60 \\
\hline Absent & 31 & 9 & 40 \\
\hline Total & 91 & 9 & 100 \\
\hline
\end{tabular}

Fisher exact test: $\mathrm{P}<0.0001$

In 60 cases of positive immunoassay test, leucocytosis was present, while in 31 cases it was absent even when test was positive. Also, leucocytosis was not there even in single case where immunoassay test was negative. Since the ' $\mathrm{P}$ ' value is $<0.05$, it can be said that there is strong association between the two.

Table 3: Association between leucocytosis and PAMG1 Immunoassay Test in study group.

\begin{tabular}{|llll|}
\hline Neonatal sepsis & PAMG1 Immunoassay & Total \\
& Positive & Negative & \\
\hline Yes & 15 & 5 & 20 \\
\hline No & 76 & 4 & 80 \\
\hline Total & 91 & 8 & 100 \\
\hline
\end{tabular}

Fisher exact test: $\mathrm{P}=0.015$

The presence of rupture of membranes was mostly associated with leucocytosis especially in cases of long standing rupture. If we compare neonatal sepsis with immunoassay test result, it is observed that sepsis was present in 15 cases of positive immunoassay test, and also in 5 cases of negative immunoassay test. The above table and $\mathrm{P}$ value $<0.05$ indicates that there is strong association between neonatal sepsis \& positive immunoassay test, which again goes in favour of long standing rupture of membranes.

\section{DISCUSSION}

PROM and its serious consequences are known common obstetric problems, finding a sensitive, specific, noninvasive and rapid test for its diagnosis is of great important. Placental alpha microglobulin-1 (PAMG-1) diagnostic test is an immunochromatography method performed in one step in which some monoclonal antibodies are used to detect the PAMG-1 proteins. PMAG-1 is a 34 kilo Dalton protein found in amniotic fluid. In order to minimise false positive or negative, two monoclonal antibodies were used to determine the sensitivity threshold level of this test.

The lowest level of PAMG-1 measured by this monoclonal antibody was 0.05 to $0.2 \mathrm{ng} / \mathrm{mL}$ from the cervicovaginal secretion. PAMG-1 found in amniotic fluid was at the range from 2,000 to $25,000 \mathrm{ng} / \mathrm{mL}$. When there was rupture of membrane, the level of PAMG-1 increased significantly in vaginal secretion. With its optimal sensitivity threshold at $5 \mathrm{ng} / \mathrm{mL}$, this test reduces the chance of faulty results. In 9 cases, the immunoassay test was negative, in 91 cases, it was positive in comparison to immunoassay test which had $100 \%$ specificity and $98.91 \%$ sensitivity, nitrazine test had $95.58 \%$ sensitivity and $100 \%$ specificity, Pooling has $97.79 \%$ sensitivity and $100 \%$ specificity, whereas ferning has $98.34 \%$ sensitivity and $100 \%$ specificity.

Also, the positive predictive value of immunoassay is $100 \%$ and chances of false positive results are minimum as compared to the conventional methods. Out of the total 91 cases detected as positive for premature rupture of membranes (either by immunoassay or 2 out of 3 clinical methods), 60 cases has marked leucocytosis, especially in long standing rupture of membranes. Neonatal sepsis is another parameter that is in a way associated with long duration of rupture of membranes and was seen in 15 out of 20 patients who had come with complaint of leaking.

When the association between pooling of liquor, a visual method and, nitrazine test and ferning test was studied, it was found that the accuracy of diagnosis of either of these 2 tests is $85 \%$. A major chunk of $15 \%$ cases is missed out by these tests and is exactly why a more accurate test to diagnose ROM is required.

If we compare the association between all the conventional methods of determination of rupture of membranes ( 2 positive out of 3 methods), with a single standard method, the sensitivity and specificity of it is poor; suggest that any single standard test will not be sufficient for the diagnosis of ROM as compared to immunoassay and its high sensitivity and specificity. In the present study, the PMAG-1 immunoassay alone has an overall sensitivity of $98.91 \%$, specificity of $100 \%$, PPV of $100 \%$, and NPV of $88.89 \%$. Hence, it is superior 
to nitrazine test or fern test alone as well as in combination (conventional standard diagnostic methods). It was also found to be accurate in the majority of women $(99.0 \%)$ that confirmed to have actual ROM later even though standard diagnostic methods were negative in some of them. The PAMG-1 immunoassay provides a quality diagnostic tool that was rapid, accurate, and with higher sensitivity and specificity compared to the other methods used currently.

Maryam Khooshideh,et al, in their study showed that PAMG-1 test showed higher sensitivity (98.9\% with $\mathrm{p}<0.001)$ and accuracy $(98 \%)$ compared with conventional tests. Marcellin et al in 80 pregnancies with or without PROM compared the diagnosis of PROM using PAMG-1 vs. IGFBP-1 in cervico-vaginal secretions. ${ }^{5,6}$

In this study, accuracy of both tests for diagnosis of PROM was the same. The sensitivity of AmniSure was also $95 \%$ and its specificity was $94.8 \%$ in this study. Abdelazim IA, et al in their study also found similar results. They also found that alkaline vagina and presence of blood have no effect on the result of the test. Birkenmaier A,et al, in their study included 199 women. Amnisure test had a sensitivity of $94.4 \%$; specificity of 98.6\%; positive predictive value, 96.2\%; negative predictive value, $98.0 \%$. In similar study by Gallot D,et al also found out that Immunoassay tests detecting Insulinlike Growth Factor-Binding Protein-1 (IGFBP-1) or Placental Alpha 1-Microglobulin (PAMG-1) are currently considered as the most useful tools for ROM diagnosis. ${ }^{7-9}$

\section{CONCLUSION}

The number of patients coming with premature rupture of membranes and preterm premature rupture of membranes in a considerable one and hence rapid and accurate test to make the diagnosis is required. The conventional methods, even though good at its own place, are not always sufficient to make the correct diagnosis. Also, the chances of false positive results are quite high.

The Placental alpha macroglobulin-1 immunoassay, is a non-invasive, rapid, one step test with very high sensitivity, specificity and accuracy as compared to the conventional methods together and also individually. Preforming this test instead of conventional methods can aid the early detection of rupture of membranes and largely affect the outcome of maternal and fetal health as timely decision can be taken once diagnosis is confirmed.

Funding: No funding sources Conflict of interest: None declared

Ethical approval: The study was approved by the Institutional Ethics Committee

\section{REFERENCES}

1. American College of Obstetricians and Gynecologists, authors.Premature Rupture of Membranes. Washington, DC: American College of Obstetricians and Gynecologists; 1998. (ACOG Practice Bulletin No.1)

2. ACOG Committee on Practice Bulletins-Obstetrics, authors. Clinical management guidelines for obstetrician-gynecologists. (ACOG Practice Bulletin No. 80: premature rupture of membranes). Obstet Gynecol. 2007;109:1007-19.

3. Cousins LM, Smok DP, Lovett SM, Poeltler DM. Amnisure placental alpha macroglobulin-1 rapid immunoassay versus standard diagnostic methods for detection of rupture of membranes. Am J Perinatol. 2005;22:317-20.

4. Boltovskaia MN, Zaraiskii EI, Fuks BB, Sukhikh GT, Kalafati TI, Starosvetskaia NA, et al. Histochemical and clinical-diagnostic study of placental alpha 1-microglobulin using monoclonal antibodies. Bull Eksp Biol Med 1991;112:397-400.

5. Maryam Khooshideh, Vida Radi, Reihaneh Hosseini, and Ladan Hosseini, The accuracy of placental alpha-microglobuline-1 test in diagnosis of premature rupture of the membranes. Iran J Reprod Med. 2015: 13(6): 355-360.

6. Marcellin L, Anselem O, Guibourdenche J, De la Calle A, Deput-Rampon C, Cabrol D, et al. Comparison of two bedside tests performed on cervicovaginal fluid to diagnose premature rupture of membranes. J Gynecol Obstet Biol Reprod (Paris) 2011;40:651-6.

7. Abdelazim IA, Makhlouf HH. Placental alpha microglobulin-1 (AmniSure test) versus insulin-like growth factor binding protein-1 (Actim PROM test) for detection of premature rupture of fetal membranes.J Obstet Gynaecol Res. 2013;39:112936.

8. Birkenmaier A, Ries JJ, Kuhle J, Burki N, Lapaire O, Hosli I. Placental-microglobulin-1 to detect uncertain rupture of membranes in a European cohort of pregnancies. Arch Gynecol Obstet. 2012;285:2125 .

9. Gallot D, Guibourdenche J, Sapin V, Goffinet F, Doret M, Langer B, et al. Which biological test to confirm rupture of membranes? J Gynecol Obstet Biol Reprod. (Paris) 2012;41:115-21.

Cite this article as: Deshpande $\mathrm{H}$, Sabale U, Madkar CS, Bobe A. A comparative study between placental alpha microglobulin-1 rapid immunoassay and standard diagnostic methods for detection of rupture of membranes. Int J Reprod Contracept Obstet Gynecol 2018;7:1813-6. 